\title{
An optimisation principle for sex allocation in a temporally varying environment*
}

\author{
Eric L. Charnov
}

Department of Biology, University of Utah, Salt Lake City, UT 84112, U.S.A.

This paper extends the theory of sex allocation to the case of temporal variation in male against female fitness options. In particular, it derives a product theorem for the phenotypic equilibrium allocation to pollen versus seeds in a cosexual plant.

Sex allocation refers to the evolution of sex ratio in dioecious species, time (and order) of sex change in sequential hermaphrodites and the allocation of resources to male versus female function in simultaneous hermaphrodites (Charnov, 1982). These problems share common features of frequency dependence of fitness (Fisher, 1930), and the phenotypic equilibrium (the ESS of Maynard Smith, 1982) may often be shown to satisfy a certain general optimality principle (Charnov, $1979 a$ ). The problem of temporal variation in male against female reproductive opportunities, and its impact on the ESS sex allocation, has been little explored (Werren and Charnov, 1978; Seger, 1983; Bull, 1981; Stubblefield, 1985). Here, I develop an ESS argument for a simultaneous hermaphrodite in a temporally varying environment; the ESS allocation will be shown to satisfy an optimality principle.

Consider the following infinite sized hermaphroditic plant population; generations are discrete and mating is at random with each individual having $R$ resources to allocate to either pollen or seed production. At a locus controlling sex allocation, let the common homozygote $(\boldsymbol{A A})$ allocate $r$ proportion of $R$ to pollen, $1-r$ to seeds. We consider the evolutionary dynamics of a rare dominant mutant $(B)$ whose bearers allocate $\hat{r}$ proportion of $R$ to pollen production. While the mutant is rare we need only consider the spread of the heterozygote $(A B)$ to know if $r$ is stable to invasion by some new sex allocation (the $\hat{r}$ ). Let $\varepsilon(T)$ be

\footnotetext{
* This paper is dedicated to Julia Laylander.
}

the frequency of $\mathrm{AB}$ at time $T, \varepsilon(T+1)$ the frequency one year (here a generation) later. Following Bull (1981) and Charnov (1979a), the following linear equation approximates the gene frequency dynamics.

$$
\varepsilon(T+1) \simeq \varepsilon(T)\left[\frac{1}{2}\left(\frac{m(\hat{r})}{m(r)}+\frac{f(\hat{r})}{f(r)}\right)\right]
$$

where $m$ and $f$ refer to the production of pollen $(m)$ and seed $(f)$ as functions of the resource input ( $r$ and $\hat{r}$ ) for the $A A$ common type and the mutant (see Charnov, 1979b, 1982; and Charnov and Bull, 1985 for further discussion of the details of production). A simple example is $m=x \cdot r^{n}$ and $f=$ $(1-r) \cdot y$ with $n, x$, and $y>0$ (Charnov, 1979b).

If the $m$ and $f$ functions do not vary through time, the ESS $r$ (Charnov et al., 1976) is that value which maximises:

$$
H=\log (m \cdot f) .
$$

It is easy to show this using relation (1). Rewriting (1) as $\varepsilon(T+1)=\lambda \cdot \varepsilon(T)$, we wish to find $r$ such that $\lambda \leqq 1$ for all $\hat{r}$ (i.e., the mutant is always selected against). For $\hat{r}$ near $r$ this can be found by the procedure of putting $\partial \lambda / \partial \hat{r}=0$, setting $\hat{r}=r$ and solving for $r$. This first order condition finds the critical point; it is an ESS provided the tradeoff of $m$ versus $f$ is convex (Charnov, 1982; Charnov et al., 1976). If we apply this product relation, equation 2 , to our simple example, $H=$ $\log \left(r^{n} \cdot(1-r) \cdot y \cdot x\right)$ and $\partial H / \partial r=0$ when $r=$ $n /(1+n)$ ( $n$ must be $<1$ for this to be a stable point). 
Note that the product relation summarises what we find in looking at $\partial \lambda / \partial \hat{r}=0$ when $\hat{r}=r$. Now, suppose that the $m$ and $f$ relations are functions of $r$ (and $\hat{r}$ ) and variables which alter through time. Returning to our simple example, $y, x$, or $n$ may change from year to year. Suppose that they are random variables whose vaues are set each year. Further suppose that the individual cannot track the temporal variation: what now is the ESS sex allocation? In an unchanging environment, the mutant sex allocation (i.e., $A B$ using $\hat{r}$ ) was selected against if $\lambda<1$; the comparable condition for our temporally varying environment is $E \log \lambda<0$ (Karlin and Liberman, 1974) where $E$ refers to expectation. I suggest that we simply apply this new stability criterion in place of the previous one; we can thus find the new ESS $r$ by putting $(\partial E \log \lambda) / \partial \hat{r}=0$, setting $\hat{r}=r$ and solving for $r$. Now, $E \log \lambda$ may be written as follows:

$$
\begin{aligned}
E \log \lambda= & \iint \cdots \int g\left(x_{1} \cdots x_{n}\right) \\
& \cdot \log \left(\frac{1}{2}\left[\frac{\hat{m}}{m}+\frac{\hat{f}}{f}\right]\right) d x_{1}, \ldots d x_{n}
\end{aligned}
$$

where $x_{i}$ refers to a temporally varying random variable which is a parameter in the $m$ and $f$ functions and where $g\left(x_{1} \ldots x_{n}\right)$ is the joint $p d f$ for the $x_{i}^{s /}$. To search for the ESS $r$ we first set $\partial E(\log \lambda) / \partial \hat{r}=0$, then set $\hat{r}=r$. This gives:

$$
\begin{aligned}
0= & \iint \cdots \int g\left(x_{1} \ldots x_{n}\right) \frac{1}{2}\left[\frac{\partial m / \partial r}{m}\right. \\
& \left.+\frac{\partial f / \partial r}{f}\right] d x_{1} \ldots d x_{n} .
\end{aligned}
$$

$B u t$, this is simply the condition*:

$$
E\left(\frac{\partial m}{m}+\frac{\partial f}{f}\right)=0 \text {. }
$$

Condition (5) is the first order condition for the ESS $r$ to maximise

$$
E[\log (m \cdot f)] .
$$

Note how this optimisation principle is simply a logical generalisation of equation 2, which applies to a temporally constant environment. To put this result in words, consider first the unchanging environment. The ESS is to maximise $\log (m \cdot f)$; this principle follows because every zygote gets half of its nuclear genes from male function $(m)$ and half from female function $(f)$.

\footnotetext{
* Equation (5) would also follow from using $E \lambda<1$ as the stability criterion (and carrying out the same differentiation).
}

In the product $m \cdot f$ male and female are treated as equivalent means for reproduction (which they are). In a temporally changing environment, we simply retain this equivalence on average.

For illustration let us apply (6) to the special case of

$$
\begin{aligned}
& m=x \cdot r^{n} \\
& f=y \cdot(1-r)
\end{aligned}
$$

where $x, y$ and $n$ are now temporally varying random variables. Now, letting a bar (-) denote average:

$$
\begin{aligned}
E \log (m \cdot f) & =E\left[\log \left(x \cdot r^{n} \cdot y \cdot(1-r)\right)\right] \\
& =\overline{\log x}+\bar{n} \log r+\overline{\log y}+\log (1-r) .
\end{aligned}
$$

Thus:

$$
\frac{\partial E \log (m \cdot f)}{\partial r}=0 \Rightarrow \frac{\bar{n}}{r}-\frac{1}{1-r}=0
$$

or

$$
r=\frac{\bar{n}}{1+\bar{n}} .
$$

Stochastic variations in $y$ and $x$ have no impact on the ESS $r$; indeed the ESS $r$ evolves to simply reflect the average $n$, quite independent of the amount of temporal variability in $n$.

This optimisation principle (just as eq. 2) requires that the hermaphroditism itself be stable, for example, against pure males or females. The deterministic theory (Charnov et al., 1976; Charnov, 1982) gives the appropriate stability conditions. For our special case, this is $n<1$ (diminishing returns on input to pollen: Charnov, 1979b). For the special case extended to include the temporal variability, the comparable condition is $\bar{n}<$ 1. Conditions for hermaphroditism with time varying parameters to be stable to invasion by pure sexes are a straightforward generalisation using $E \log \lambda<0$ in place of $\lambda<1$.

It is simple to extend these hermaphrodite results to encompass factors affecting male versus female reproductive success in addition to production of gametes; for example, time variation in pollinating agents which must carry pollen away if it is to gain fertilisations (Charnov and Bull, 1985). This brief note develops the basic generalisation $[\max : E \log (m \cdot f)]$ independent of these added complications.

As a final question, we may ask just what reproductive factors would be expected to show time variation; recall that it is time variation in male compared to female. For instance, if total plant 
reproductive resource $(R)$ changes from year to year but this does not affect, in our simple example, the shape of the male gain relation (does not change $n$ ), such variation does not affect sex allocation. At least three quite changeable things may affect sex allocation. First, total plant resource $(R)$ which, contrary to the above illustration, could well be expected to alter the male gain relation (i.e., change $n$ ). Second, time variation in availability of pollinating agents which ought also to affect $n$ (also perhaps $x$ and $y$; but note that this does not affect the ESS allocation). Thirdly, seed dispersal agents may vary in effectiveness from year to year and alter $f$ (see Charnov and Bull, 1985 for further development).

It is surprising that the ESS result in equation 7 contains only the time average of the variable $n$; while the power model (i.e., $m \propto r^{n}$ ) is specific, the shape parameter $n$ allows a great many shapes to be discussed. Other models for $m$ and $f$ should be studied but my guess is that in general temporal variance in shape will play a minor role and the average shape will dominate the ESS.

Acknowledgments Conversations with J. J. Bull were of much help in thinking about these ideas. Robin Baker helped me to expand the biological discussion.

\section{REFERENCES}

BULL, J. J. 1981. Sex ratio when fitness varies. Heredity, 46, 9-26. CHARNOV, E. L. $1979 a$. The genetical evolution of patterns of sexuality: Darwinian fitness. Amer. Natur., 113, 465-480.

CHARNOV, E. L. $1979 b$. Simultaneous hermaphroditism and sexual selection. Proc. Natn. Acad. Sci. USA, 76, 2480-2484.

Charnov, E. L. 1982. The Theory of Sex Allocation. Princeton Univ. Press, Princeton, N.J., USA.

CHARNOV, E. L. AND BULL, J. J. 1985. Sex allocation and pollinator attraction in cosexual plants. Jour. Theor. Biol., (in press).

CHARNOV, E. L., MAYNARD SMITH, J. AND BULL, J. J. 1976. Why be an hermaphrodite? Nature, 263, 125-126.

FISHER, R. A. 1930. The Genetical Theory of Natural Selection (reprint 1958). Dover, N.Y.

KARLIN, S. AND LIEBERMAN, U. 1974. Random temporal variation in selection intensities: case of large population sizes. Theor. Pop. Biol., 6, 355-382.

MAYNARD SMITH, J. 1982. Evolution and the Theory of Games. Cambridge Univ. Press, Cambridge, U.K.

SEGER, J. 1983. Partial bivoltinism may cause alternating sexratio biases that favor eusociality. Nature, 301, 59-62.

STUBBLEFIELD, J. W. 1985. Sex allocation in bivoltine populations: Unbeatable investment ratios and implications for the origin of eusociality. (Submitted to Jour. Theor. Biol.)

WERREN, J. H. AND CHARNOV, E. L. 1978. Facultative sex ratio and population dynamics. Nature, 272, 349-350. 